\title{
Volatile Constituents of Trigonella stellate
}

\author{
Tariq R. Sobahi \\ Department of Chemistry, Faculty of Science, \\ King Abdulaziz University, Jeddah, Saudi Arabia
}

\begin{abstract}
In this article, the volatile constituents of Trigonella stellate collected from Wadi Qattan, northern east Hazn mountain about 130 $\mathrm{km}$ south of Taief, Saudi Arabia, using GC/MS are described. In addition to many fat derivatives, $\beta$-sitosterol and stigmasterol, about twenty two volatile compounds were identified. This Trigonella species has not been investigated previously for its natural products.
\end{abstract}

\section{Introduction}

Literature survey indicated that Trigonella stellate is only screened for flavonoids and saponins ${ }^{[1]}$, but no chemical investigations have been done on the volatile constituents for this species.

From a medicinal point of view, Trigonella species were found to be important. Kaur and Kapoor ${ }^{[2]}$ and Langmead et al. ${ }^{[3]}$ studied the antioxidant activity of Trigonella species. The protective effects of total saponins of Trigonella foenum-graecum on acute cerebral ischemia were studied by Li and coworkers ${ }^{[4]}$.

Typical chemical constituents of the genus Trigonella are diosgenin ${ }^{[5]}$, furostanol type steroidal saponins ${ }^{[6]}$, flavonoids ${ }^{[7]}$ and flavonol glycosides ${ }^{[8]}$.

\section{Experimental}

GC/MS spectra were taken on QP-7000 Shimadzu, with a fused silica capillary column ( $30 \mathrm{~m} \times 0.25 \mathrm{~mm}$ ID), film ( $5 \%$ phenyl, $95 \%$ methylsilicon) thickness $0.25 \mu$, and the output is an IBM computer with software class 5000 and NIST library for comparison. 


\section{The Plant Material}

The aerial parts of Trigonella stellate, in the flowering stage, are collected from Wadi Qattan, northern east Hazn mountain about $130 \mathrm{~km}$ south of Taief, in April 2003 and identified by Dr. F. Alghamdi, Botany Dept., Faculty of Science, King Abdulaziz University. A specimen was deposited in the Herbarium of Botany Dept., Faculty of Science, King Abdulaziz University.

\section{Processing of Plant Material}

The air-dried, ground aerial parts $(300 \mathrm{~g})$ were extracted at room temperature by soaking in a mixture $1: 1: 1$ of methanol/ether/pet. ether $40-60^{\circ}$ for 24 hours. The crude extract $(6.5 \mathrm{~g})$ was defatted by dissolving in cold $\mathrm{MeOH}(50 \mathrm{ml})$ and standing in the fridge freezer for overnight, then, quick filtration and evaporation gave the defatted extract $(3.8 \mathrm{~g})$. The defatted extract was fractionated over silica gel $(100 \mathrm{~g}) \mathrm{CC}(100 \mathrm{~cm}$ length $\times 4 \mathrm{~cm}$ inner diameter) using stepwise elution into five fractions. Fr3a (15.8\%) and Fr3b (10.5\%) using the eluent mixture pet. ether/ether 3:1; Fr4 (32.4\%) using pet. ether/ether 1:1; Fr5a (11.8\%) using ether and $\mathrm{Fr} 5 \mathrm{~b}$ (10.3\%) using ether/MeOH 9:1.

\section{Identification of Compounds}

Fraction Fr3a afforded by GC/MS lauric acid $\left(R_{t} 16.20 \mathrm{~min}, 0.07 \%\right)$, myristic acid $\left(R_{t} 18.56 \mathrm{~min}, 0.42 \%\right)$, octadecane $\left(R_{t} 18.91 \mathrm{~min}, 0.34 \%\right)$, methylpentadecanoate $\left(R_{t} 19.19 \mathrm{~min}, 0.08 \%\right)$, pentadecanoic acid $\left(R_{t} 19.64 \mathrm{~min}\right.$, $0.44 \%)$, methyl palmitate $\left(R_{t} 20.28 \mathrm{~min}, 3.37 \%\right),(Z)$ phytol $\left(R_{t} 20.50 \mathrm{~min}\right.$, $0.36 \%)$, palmitic acid $\left(R_{t} 21.01 \mathrm{~min}, 27.57 \%\right)$ methyl heptadecanoate $\left(R_{t} 21.30\right.$ min, $0.47 \%)$, heptadecanoic acid $\left(R_{t} 21.76 \mathrm{~min}, 1.10 \%\right)$, methyl linoleate $\left(R_{t}\right.$ 21.99 min, $1.79 \%)$, (E) phytol ( $\left.R_{t} 22.19 \mathrm{~min}, 4.49 \%\right)$, methyl stearate $\left(R_{t} 22.29\right.$ min, $0.85 \%)$, stearic acid $\left(R_{t} 22.82 \mathrm{~min}, 7.28 \%\right)$, nonadecanoic acid $\left(R_{t} 23.60\right.$ min, $1.05 \%)$, methyl eicosanoate $\left(R_{t} 24.14 \mathrm{~min}, 1.36 \%\right)$, arachidic acid $\left(R_{t}\right.$ $24.52 \mathrm{~min}, 6.52 \%)$, methyl heneicosanoate $\left(R_{t} 25.00 \mathrm{~min}, 0.73 \%\right)$, heneicosanoic acid $\left(R_{t} 25.37 \mathrm{~min}, 0.91 \%\right)$ and docasanoic acid $\left(R_{t} 26.41 \mathrm{~min}, 2.80 \%\right)$.

Fraction Fr3b gave by GC/MS 4-hydroxy-3-methoxyacetophenone $\left(R_{t} 8.00\right.$ min, $0.76 \%)$, vanillic acid $\left(R_{t} 9.15 \mathrm{~min}, 3.12 \%\right)$, dehydronerol $1\left(R_{t} 10.00 \mathrm{~min}\right.$, $0.26 \%)$, 4-hydroxy-3,5-dimethoxyacetophenone $\left(R_{t} 11.16 \mathrm{~min}, 0.17 \%\right)$, palmitic acid $\left(R_{t} 13.67 \mathrm{~min}, 4.89 \%\right),(Z)$ phytol $\left(R_{t} 15.18 \mathrm{~min}, 1.13 \%\right)$ and stearic acid $\left(R_{t} 15.65 \mathrm{~min}, 0.74 \%\right)$.

Fraction Fr4 gave by GC/MS terpin-4-ol $\left(R_{t} 3.44 \mathrm{~min}, 1.91 \%\right), \alpha$-terpineol $\left(R_{t} 3.62 \mathrm{~min}, 1.96 \%\right)$, citronellol $2\left(R_{t} 4.15 \mathrm{~min}, 3.28 \%\right), E$-citral $\left(R_{t} 4.34 \mathrm{~min}\right.$, $2.30 \%)$, geraniol $\left(R_{t} 4.56 \mathrm{~min}, 2.17 \%\right)$, dihydromyrcene $3\left(R_{t} 6.06 \mathrm{~min}, 1.92 \%\right)$, 
eugenol $\left(R_{t} 6.14 \mathrm{~min}, 2.09 \%\right)$, geranyl acetate $\left(R_{t} 6.52 \mathrm{~min}, 1.71 \%\right)$, eugenol methyl ether $\left(R_{t} 6.82 \mathrm{~min}, 1.63 \%\right), Z$-caryophyllene $4\left(R_{t} 7.11 \mathrm{~min}, 1.84 \%\right), \alpha-$ humulene 5 ( $\left.R_{t} 7.59 \mathrm{~min}, 1.77 \%\right)$, caryophyllene D $6\left(R_{t} 7.98 \mathrm{~min}, 1.91 \%\right), \alpha-$ bulnesene 7 ( $\left.R_{t} 8.32 \mathrm{~min}, 1.84 \%\right)$, cubenol $8\left(R_{t} 10.25 \mathrm{~min}, 0.61 \%\right)$, 8heptadecene $\left(R_{t} 10.47 \mathrm{~min}, 1.95 \%\right)$, farnesol $\left(R_{t} 11.02 \mathrm{~min}, 1.91 \%\right)$, 4-hydroxy3,5-dimethoxyacetophenone $\left(R_{t} 11.17 \mathrm{~min}, 1.71 \%\right)$, palmitic acid $\left(R_{t} 13.66 \mathrm{~min}\right.$, $2.61 \%)$, aesculetin dimethyl ether $9\left(R_{t} 13.78 \mathrm{~min}, 2.28 \%\right),(Z)$ phytol $\left(R_{t} 15.17\right.$ min, 0.97\%) and campesterol ( $R_{t} 22.19$ min, 16.04\%). Fraction Fr5a afforded $\beta$ sitosterol and stigmasterol (1:1) (identified by their ${ }^{1} \mathrm{H}-\mathrm{NMR}$ spectra).

Fraction Fr5b afforded by GC/MS benzoic acid $\left(R_{t} 5.43 \mathrm{~min}, 0.38 \%\right)$, 4hydroxy-3,5-dimethoxyacetophenone $\left(R_{t} 13.21 \mathrm{~min}, 0.03 \%\right)$, coniferyl alcohol $\left(R_{t} 13.27 \mathrm{~min}, 0.09 \%\right)$, myristic acid $\left(R_{t} 13.53 \mathrm{~min}, 0.08 \%\right)$, 2,3,6,7-tetrahydrofarnesol $\left(R_{t} 14.38 \mathrm{~min}, 0.11 \%\right), 6,10$-dimethyl-2-undecanone $\left(R_{t} 14.14\right.$ min, $0.14 \%)$, pentadecanoic acid $\left(R_{t} 14.63 \mathrm{~min}, 0.05 \%\right)$, methyl palmitate $\left(R_{t}\right.$ $15.29 \mathrm{~min}, 0.24 \%)$, palmitic acid $\left(R_{t} 15.72 \mathrm{~min}, 5.23 \%\right)$, heptadecanoic acid $\left(R_{t}\right.$ $16.68 \mathrm{~min}, 0.11 \%)$, $(Z)$ phytol $\left(R_{t} 17.18 \mathrm{~min}, 2.65 \%\right)$, stearic acid $\left(R_{t} 17.66 \mathrm{~min}\right.$, $1.07 \%)$, arachidic acid $\left(R_{t} 19.48 \mathrm{~min}, 0.59 \%\right)$ and campesterol $\left(R_{t} 36.70 \mathrm{~min}\right.$, $1.44 \%)$.

\section{Results and Discussion}

Fractions described above were found, by ${ }^{1} \mathrm{H}-\mathrm{NMR}$ to contain complicated mixtures of weakly polar components. Therefore, GC/MS technique was subsequently used to analyze the constituents of these fractions. Many compounds were identified based upon the comparison of the mass spectrum given by the computer from the NIST library with the actual spectrum of each component. After that, the structure was further ascertained by comparing their mass spectral data with those available in Adams ${ }^{[9]}$ or of the corresponding compound from the literature.

In addition to many fatty acids and fatty acid derivatives, about six shikimate derivatives, eight monoterpenes and six sesquiterpenes were identified from the species under investigation, Trigonella stellate, which has not been previously investigated for its volatile components. 
<smiles>CC(C)=CC=CC(C)=CCO</smiles><smiles>C=C1CCC/C(C)=C/CCC2CC1C(C)(C)C2</smiles>

4

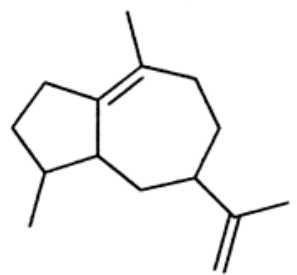

7<smiles>CC(C)=CCCC(C)CCO</smiles>

2<smiles>C/C1=C\CC/C(C)=C/C/C(C)=C/C1</smiles>

5<smiles>CC1=C[C@H]2C(C(C)C)CC[C@@H](C)[C@]2(O)CC1</smiles>

8<smiles>CC=C(C)CCC=C(C)C</smiles>

3<smiles>C=C1/C=C/C(C(C)C)CC/C(C)=C/CCC1=C</smiles>

6<smiles>COc1cc2ccc(=O)oc2cc1OC</smiles>

9

Scheme 1

\section{References}

[1] Rizk, A.M., Heiba, H.I., MáAyergi, H.A. and Batanouny, K.H., Fitoterapia, 57: 3 (1986).

[2] Kaur, C. and Kapoor, H.C., International J. Food Sci. Tech., 37: 153 (2002).

[3] Langmead, L., Dawson, C., Hawkins, C., Banna, N., Loo, S. and Rampton, D.S., Alimmentary Pharm. Ther., 16: 197 (2002).

[4] Li, L., Ran, X., Mao, X., Wang, X., Zhang, J. and Wang, F., Zhongguo Yaolixue Tongbau, 17: 92 (2001).

[5] Huang, W., Duan, J. and Yang, L., Zhongguo Zhongyao Zazhi, 24: 595 (1999).

[6] Murakam, T., Kishi, A., Matsuda, H. and Yoshikawa, M., Chem. Pharm. Bull., 48: 994 (2000).

[7] Yuldashev, M.P., Khim. Prir. Soedin., 38: 291 (2002).

[8] Han, Y., Nishibe, S., Noguchi, Y. and Jin, Z., Phytochemistry, 58: 577 (2001).

[9] Adams, R.P., "Identification of Essential Oil Components by GC/MS", Allured Publishing Corporation, Carol Stream, Illinois, USA (1995). 


\title{
المكونات الكيميائية لنبات السطيح ( ترايجونيلا ستيلات )
}

\author{
طارق رشاد سبحي \\ قسم الكيمياء ، كلية العلوم ، جامعة الملك عبد العزيز

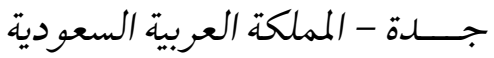

المستـخلص. في هذا البـحث ، تم جـمع نبـات السطيح " ترايجـونيلا

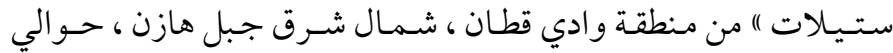

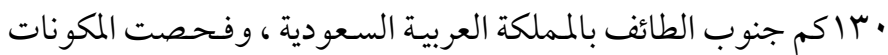

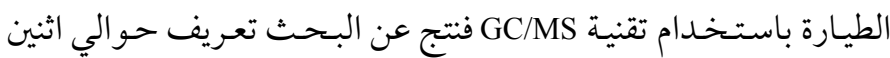

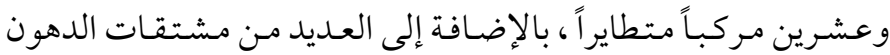

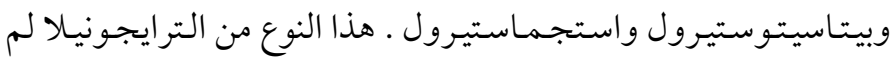

يفحص محتواه من المنتجات الطبيعية من قبل. 